\title{
Relevance of the novel IASLC/ATS/ERS classification of lung adenocarcinoma in advanced disease
}

\author{
Alma D. Campos-Parra1', Alejandro Avilés², Susana Contreras-Reyes', \\ Carlos E. Rojas-Marín ${ }^{3}$, Roberto Sánchez-Reyes ${ }^{1}$, Rafael J. Borbolla-Escoboza ${ }^{4}$ \\ and Oscar Arrieta ${ }^{1}$
}

Affiliations: ${ }^{1}$ Thoracic Oncology Unit and Experimental Oncology Laboratory, Instituto Nacional de Cancerología (INCan), México City, ${ }^{2}$ Dept of Pathology, Instituto Nacional de Cancerología (INCan), México City, ${ }^{3}$ Dept of interventional radiology, Instituto Nacional de Cancerología (INCan), México City, ${ }^{4}$ Oncology, Bristol-Myers-Squibb, México, Mexico City, Mexico.

Correspondence: O. Arrieta: Thoracic Oncology Unit and Experimental Oncology Laboratory of Instituto Nacional de Cancerología (INCan), San Fernando \#22, Col. Sección XVI, Tlalpan, 14080 Mexico City, Mexico: E-mail: ogardunam.mx

ABSTRACT Since the International Association for the Study of Lung Cancer (IASLC), American Thoracic Society (ATS) and European Respiratory Society (ERS) reported a new lung adenocarcinoma (ADC) classification, several groups have validated its association with prognosis in early stage disease. To our knowledge, there are no studies in advanced disease.

We reviewed 313 patients with invasive lung ADC who were re-classified using the new IASLC/ATS/ERS criteria. Patients received platinum-based chemotherapy. Clinical characteristics, EGFR mutations, response and progression-free survival (PFS) after chemotherapy and overall survival were analysed.

ADCs were classified as lepidic $7.4 \%$, acinar $44.7 \%$, papillary $10.1 \%$, micropapillary $3.5 \%$ and solid $34.2 \%$. When patterns were lumped into groups, response rates and PFS to platinum-based chemotherapy were better in high-grade ADC (micropapillary, papillary and solid-predominant) versus intermediate-grade ADC (lepidic and acinar-predominant) (36.9\% versus $25.4 \% \mathrm{p}=0.034$ and 6.4 versus 5.5 months $\mathrm{p}=0.009$, respectively). Overall survival was better in high-grade ADC ( 25 versus $16.8 ; \mathrm{p}=0.023)$. Factors associated with better overall survival were Eastern Cooperative Oncology Group (0-1), EGFR mutations and highgrade ADC.

Prognostic differences found with the new classification in early disease may not apply to patients with advanced disease. Unlike in early stages, patients with high-grade ADC have longer overall survival compared with intermediate-grade ADC, probably due to a better response to chemotherapy.

@ERSpublications

Impact of the novel classification of lung adenocarcinoma in advanced disease http://ow.ly/rJBdG

For editorial comments see page 1240.

Received: Aug 092013 | Accepted after revision: Nov 272013 | First published online: Jan 162014

Clinical trial: This study is registered at ClinicalTrials.gov with identifier number NCT01023828.

Conflict of interest: None declared.

Copyright @ERS 2014 


\section{Introduction}

Lung cancer is the leading cause of cancer-related mortality worldwide [1]. Mean annual cancer-related mortality in Mexico from 1998-2004 was 397400 deaths; 45578 of those deaths were due to lung cancer [2,3]. Non-small cell lung cancer (NSCLC) accounts for around 85\% of all lung cancers and consists of several subtypes: adenocarcinoma (ADC) (85-90\%), squamous-cell lung cancer (15-20\%) and large-cell lung cancer $(1-5 \%)$. The rates of ADC are increasing in most countries and most patients are diagnosed in advanced disease (USA 84\% and Mexico 98\%) [4].

A new lung ADC classification has been proposed by the International Association for the Study of Lung Cancer (IASLC), American Thoracic Society (ATS) and European Respiratory Society (ERS) using comprehensive histological subtyping with lepidic, acinar, papillary and solid patterns; micropapillary was added as a new histological subtype [5]. The relevance of this new ADC classification resides in that it may have differences in clinicopathological features and prognosis as well as in the different risk factors associated with lung cancer, which may impact the adjuvant and palliative treatment decisions. Potentially, this classification could help to distinguish subgroups of patients who may benefit from adjuvant in early disease and possibly identify subgroups with better response to palliative treatment.

The prognostic impact of the novel IASLC/ATS/ERS classification of pulmonary ADC has previously been validated in US cohorts of stage I tumours for disease-free survival (DFS). Three overall prognostic groups were identified: low grade that included ADC in situ and minimally invasive; intermediate grade that included nonmucinous lepidic predominant, papillary predominant and acinar predominant; and high grade that include invasive mucinous, colloid predominant, solid predominant and micropapillary predominant [6]. Eventually, one smaller Australian cohort of stage I, II, and III tumours, a German cohort and Japanese cohort of patients who underwent resection, confirmed that the new subtypes of ADCs can be classified according to prognosis [7-9]. However, to our knowledge, there are no studies describing the potential impact of the classification on prognosis in advanced disease, which corresponds to the majority of patients worldwide.

\section{Patients and methods}

\section{Patient selection}

We retrospectively evaluated patients with invasive lung ADC (stages IIIB and IV) seen at the Lung Cancer Clinic in Mexico's National Cancer Institute from 2007-2010. Initial classification was made according to the current WHO classification for lung cancer [10], and was later re-classified according to the novel IASLC/ATS/ERS proposal [11]. Tumour tissue from all patients was evaluated by immunohistochemistry, which was consistent with histopathological ADC diagnosis. In all patients, the tissue was obtained by computed tomography-guided Tru-Cut (Carefusion, San Diego, CA, USA) biopsy. All samples were reanalysed by experts and pathologists blinded to the clinical characteristics and prognosis of patients.

A complete medical history including a detailed history of smoking, smoke exposure and physical examination was obtained; tumour specimens were collected at the time of diagnosis. Wood smoke exposure (WSE) was defined as being exposed to fumes resulting from burning wood in fireplaces and wood stoves for $\geqslant 4 \mathrm{~h} \cdot \mathrm{day}^{-1}$ for $>5$ years. A nonsmoker was defined as being someone having a lifetime exposure of fewer than 100 cigarettes, the tobacco smoking index was calculated by multiplying the number of cigarette packs consumed per day by the number of years spent smoking. This study was approved by our institution's review board and ethics committee as a part of the report "Clinical and Pathological Characteristics, Outcome and Mutational Profiles Regarding Non-Small-Cell Lung Cancer Related to Wood-Smoke Exposure" registered with ClinicalTrials.gov: NCT01023828.

\section{Treatment regimen}

All patients were treated according to international guidelines for the treatment of lung cancer [12]. All received platinum-based chemotherapy as first line of treatment. Chemotherapy regimens included paclitaxel plus cisplatin or carboplatin, and vinorelbine plus cisplatin or carboplatin. Only 16\% of all patients received epidermal growth factor receptor (EGFR) tyrosine-kinase inhibitor (TKI) (gefitinib or erlotinib) as the second or third line of treatment, after progression to cytotoxic chemotherapy because the mutational results were obtained retrospectively. Patients with cytological diagnosis and without enough tissue for immunohistochemistry or to detect the histological pattern were not evaluated.

\section{DNA extraction and mutational analysis}

All biopsies were taken using chemotherapy-guided Tru-Cut and were analysed by the pathology department for their histological diagnosis and neoplastic cellularity quantification $(>50 \%)$; they were later embedded in paraffin until processing for DNA extraction. Genomic DNA was extracted from areas of 
paraffin slides using a standard procedure and a QIAamp DNAFFPE tissue kit (QIAGEN, Hilden, Germany), according to the manufacturer's instructions. EGFR (exons 18, 19, 20 and 21) gene mutations were detected by therascreen RGQ PCR kit (QIAGEN, using the Scorpions ARMS method) which combines ARMS and Scorpions technologies for detecting mutations in real-time PCR reactions. Real-time PCR was performed using a Rotor-Gene Q 5plex HRM (QIAGEN), according to the manufacturer's instructions. We classified mutations as "common" or "uncommon".

\section{Statistical analysis}

Continuous variables were summarised as arithmetic means, medians and standard deviations for descriptive purposes and categorical variables were expressed as percentages with $95 \%$ confidence intervals (95\% CI). An unpaired t-test or Mann-Whitney U-tests were used for inferentially comparing two groups, according to data distribution determined by Kolmogorov-Smirnov test. Chi-squared or Fisher's exact test were used for assessing categorical variable significance. Statistical significance was determined as $\mathrm{p} \leqslant 0.05$ using a two-tailed test. Statistically significant and borderline significant variables $(\mathrm{p}<0.1)$ were included in multivariate logistic regression analysis. Overall survival was measured from day of diagnosis to date of death or last follow-up visit and analysed by Kaplan-Meier technique; subgroups were compared using the log-rank test. All variables were dichotomised for survival curve analysis. Cox multivariate analysis was used for adjusting for potential confounders. Data were analysed using SPSS software package, version 15 (SPSS, Inc., Chicago, IL, USA).

\section{Results}

\section{Clinical characteristics}

257 patients met the histological criteria for inclusion. Most patients were females (59.7\%), cigarette smoking was present in 50.5\% of patients and $42.9 \%$ had WSE. Clinical stage distribution (as per the American Joint Committee on Cancer 2010) was $80.1 \%$ stage IV and $19.8 \%$ stage III. $74.6 \%$ had an Eastern Cooperative Oncology Group (ECOG) performance status of 0 or 1 . The most frequent metastasis site was lung $(41.3 \%)$ and the most frequent symptom was cough $(66.3 \%)$ (table 1$)$.

\section{Clinical and pathological characteristics associated with the adenocarcinoma subtypes of the IASLC/ ATS/ERS classification}

According to the new IASLC/ATS/ERS classification; 7.4\% were lepidic-predominant ADC, $44.7 \%$ acinarpredominant ADC, $10.1 \%$ papillary-predominant ADC, 3.5\% micropapiliary-predominant ADC and $34.2 \%$ solid-predominant ADC (table 2). The histological types were distributed into two prognostic groups according to grade as follows: intermediate-grade ADCs, corresponding to lepidic-predominant ADC and acinar-predominant ADC (52.1\%); and high-grade ADCs, corresponding to micropapillary-predominant ADC, solid-predominant ADC and papillary-predominant ADC (47.8\%) [7]. Table 1 shows that the histology classification did not correlate with age, sex, ECOG, cigarette smoking or wood smoke exposure. Nevertheless, in the high-grade ADC group, lymph metastases $(\mathrm{p}=0.027)$ and thoracic $\mathrm{pain}(\mathrm{p}=0.029)$, were more frequent.

\section{EGFR mutation associated with the IASLC/ATS/ERS classification}

EGFR activating mutations were detected in $41.8 \%$ of all patients. The mutations detected were: in exon 18 (6.5\%); deletions in exon 19 (46.3\%); in exon 20, the point mutations S768I (2.6\%) and T790M (6.5\%); and, in exon 21, the L858R point mutation (33.3\%). The most common mutation was in-frame deletion in exon 19 and the second most common mutation was point mutation in exon 21 (L858R). EGFR mutations were more frequently in females $(50$ versus $19(\mathrm{p}=0.018)$ ), in never-smoking patients (52 versus 17 $(\mathrm{p}<0.001))$ and in patients with WSE $(43$ versus $26(\mathrm{p}<0.001))$. Table 3 shows that the EGFR mutations were not associated with any subgroup in the IASLC/ATS/ERS classification. Of the patients that harbouring EGFR mutations, the $79.5 \%$ presented common EGFR mutations and only the $20.5 \%$ presented uncommon mutations $(p=0.01)$. Lepidic and acinar adenocarcinoma histologic subtypes were associated with a greater frequency of EGFR common mutations $(p=0.01)$.

\section{Response rate to chemotherapy}

Overall response rate to platinum-based chemotherapy was $31.8 \%$ (1.1\% complete response and $30.3 \%$ partial response); stable disease was seen in $44.4 \%$ of patients and the disease progressed in $23.7 \%$. Interestingly, we found a higher response rate in high-grade group compared with the intermediate-grade group (36.9\% (95\% CI 30.9-42.9) versus $25.4 \%$ (20-30.8); $\mathrm{p}=0.034)$. Multivariate analysis showed that the only factor associated with better response was stage III compared with stage IV (49.1 versus $27.2 ; \mathrm{p}=0.002)$. 
Progression-free survival after chemotherapy

Median progression-free survival (PFS) after chemotherapy was 5.9 months. According to our two prognostic groups, it was significantly better for the high-grade patients compared with those in the intermediate-grade (6.4 (5.0-7.9) versus 5.5 (4.6-6.5) months; $\mathrm{p}=0.009)$ (fig. 1a). In the multivariate analysis presence of ECOG $0-1$ and tumour stage III was associated with significant improvement in PFS after chemotherapy (table 4).

\section{Overall survival}

Overall survival was 20.7 months (95\% CI 16.2-25.3). The overall survival was better for patients in the high-grade group than for those in intermediate-grade prognosis group (25 (15.4-34.6) versus 16.8 $(11.8-21.8)$ months; $p=0.023)$ (fig. 1b). Factors associated with significantly longer overall survival were ECOG of 0-1 (HR $2.04(1.168-3.56) ; \mathrm{p}=0.012)$, stage III (HR $2.16(1.08-4.30) ; \mathrm{p}<0.029)$, presence of EGFR mutations (HR $1.99(1.142-3.498) ; \mathrm{p}<0.015)$ and high-grade histology (HR 0.804 (0.634-1.036); $\mathrm{p}=0.037)($ table 5).

\section{Discussion}

The IASLC/ATS/ERS reported a new classification for lung ADC and several cohorts of early stages have validate their association with prognosis, in US cohorts of stage I tumours, the prognostic impact was analysed, in one smaller Australian cohort of stage I-III tumours, in a German cohort of stages I-IV (although only 2.3 years, $3.0 \%$ in stage IIIB and IV) and in a German and Japanese cohort of patients who

\section{TABLE 1 Characteristics of all patients according to predominant subtype}

\section{All patients \\ Intermediate group} (lepidic and acinar)
High group (papillary, micropapillary and solid) p-value 123 (39.3)

$63(51.2)$

$60(48.8)$

$77(62.6)$

$46(37.4)$

$90(73.6)$

$33(26.4)$

$33(26.4)$

$90(73.6)$

$67(54.5)$

$56(45.5)$

73 (59.0)

50 (41.0.)

$57(46.3) \quad 0.132$

$30(24.4) \quad 0.539$

$33(26.8) \quad 0.888$

$13(10.6) \quad 0.207$

$33(26.8) \quad 0.027$

6(4.9) 0.878

$37(30.1) \quad 0.572$

$64(52.0) \quad 0.353$

$83(67.5) \quad 0.111$

$55(44.7) \quad 0.850$

$15(12.2) \quad 0.364$

$60(48.8) \quad 0.029$

Data are presented as $\mathrm{n}$ or $\mathrm{n}(\%)$, unless otherwise stated. ECOG: Eastern Cooperative Oncology Group; CNS: central nervous system. 
TABLE 2 Adenocarcinoma subtypes according to the International Association for the Lung Cancer, American Thoracic Society and European Respiratory Society classification

Invasive adenocarcinoma

Lepidic predominant

Acinar predominant

Papillary predominant

Micropapillary predominant

Solid predominant

Not applicable n $(\%)$

$19(6.1)$

$115(36.7)$

$26(8.3)$

$9(2.9)$

$88(28.1)$

$56(17.9)$

Total number of patients was 313 .

underwent resection [6-10]. To our knowledge, no studies have validated the prognosis impact of the new classification in advanced disease, which corresponds to the majority of patients worldwide and in our country, Mexico.

In our results, papillary-predominant $\mathrm{ADC}$ was present in $10.1 \%$ of cases, this frequency is similar to Japanese and German studies [8,9]; however, the US cohort has a high frequency of $28 \%$ [6], this may be because to the heterogeneity of geographical areas with different risk factors or histology or international observational variability among pathologists.

\begin{tabular}{|c|c|c|c|}
\hline & Wildtype & EGFR mutated & p-value \\
\hline Patients ${ }^{\#}$ & $96(58.2)$ & $69(41.8)$ & \\
\hline \multicolumn{4}{|l|}{ Age } \\
\hline$<60$ years & 52 (54.2) & $33(47.1)$ & \multirow[t]{2}{*}{0.230} \\
\hline$>60$ years & $44(45.8)$ & $36(52.9)$ & \\
\hline \multicolumn{4}{|l|}{ Sex } \\
\hline Female & $53(55.2)$ & 50 (72.5) & \multirow[t]{2}{*}{0.018} \\
\hline Male & $43(44.8)$ & 19 (27.5) & \\
\hline \multicolumn{4}{|l|}{ ECOG } \\
\hline $0-1$ & 69 (71.9) & $49(72.1)$ & \multirow[t]{2}{*}{0.562} \\
\hline $2-4$ & $27(28.1)$ & 20 (27.9) & \\
\hline \multicolumn{4}{|l|}{ Tumour stage } \\
\hline III & 19 (19.8) & 10 (13.2) & \multirow[t]{2}{*}{0.188} \\
\hline IV & 77 (80.2) & $59(86.8)$ & \\
\hline \multicolumn{4}{|l|}{ Smoking status } \\
\hline Positive & $52(54.2)$ & $17(24.6)$ & \\
\hline Negative & $44(45.8)$ & $52(75.4)$ & $<0.001$ \\
\hline \multicolumn{4}{|l|}{ Wood smoke exposure } \\
\hline Positive & 31 (32.3) & $43(61.8)$ & \multirow[t]{2}{*}{$<0.001$} \\
\hline Negative & 65 (67.7) & $26(38.2)$ & \\
\hline \multicolumn{4}{|l|}{ Histological subtypes } \\
\hline Lepidic predominant & $4(50.0)$ & $4(50.0)$ & \multirow[t]{6}{*}{0.665} \\
\hline Acinar predominant & 35 (58.3) & $25(41.7)$ & \\
\hline Papillary predominant & $8(50.0)$ & $8(50.0)$ & \\
\hline Micropapillary predominant & $3(75.0)$ & $1(25.0)$ & \\
\hline Solid predominant & $34(66.7)$ & $17(33.3)$ & \\
\hline NA & 12 (46.2) & $14(53.8)$ & \\
\hline \multicolumn{4}{|l|}{ Groups } \\
\hline Intermediate & 39 (57.4) & $29(42.6)$ & \multirow[t]{3}{*}{0.308} \\
\hline High & 45 (63.4) & $26(36.6)$ & \\
\hline NA & 12 (46.2) & 14 (53.8) & \\
\hline
\end{tabular}

Data are presented as $\mathrm{n}(\%)$, unless otherwise stated. ${ }^{\#}$ : total number of patients was 145. EGFR: epidermal growth factor receptor; ECOG: Eastern Cooperative Oncology Group; NA: not applicable. 

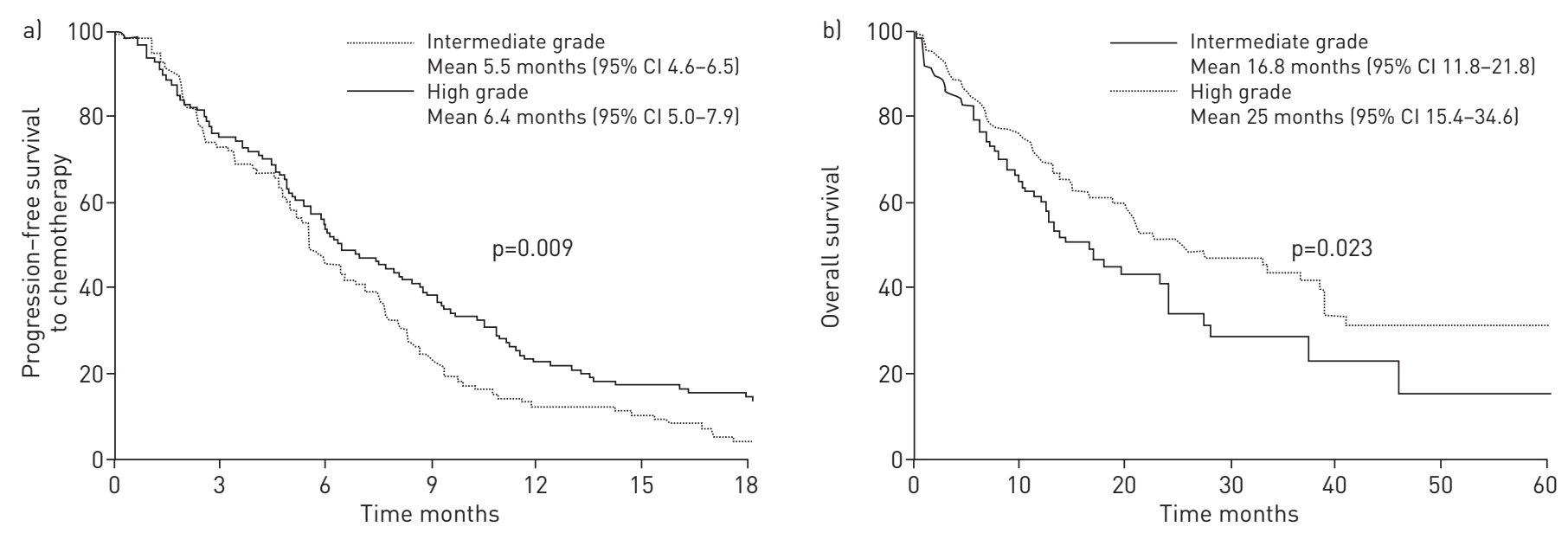

FIGURE 1 Progression-free survival to chemotherapy (a) and overall survival (b) in intermediate and high grade prognostic groups.

According to early disease, a US study found that, in untreated stage I and intermediate-grade ADC patients, the 5-year DFS was $83-90 \%$, but in high-grade histology patients it was $67-70 \%(\mathrm{p}<0.001)$ [6]. Similarly, an Australian study, reported that in patients who had primary intermediate-grade group lung cancer who were resected and with curative intent had 60-71\% 5-year DFS, although high-grade patients had only 38\% 5-year DFS [7]. A Japanese study also supported these findings suggesting that the new classification is an independent predictor of DFS, in patients that undergo resection and within the intermediate and high-risk group [9].

TABLE 4 Factors associated with progression-free survival to chemotherapy

\begin{tabular}{|c|c|c|c|c|c|}
\hline & \multirow{2}{*}{$\begin{array}{l}\text { Progression-free } \\
\text { survival months }\end{array}$} & \multirow{2}{*}{$\begin{array}{l}\text { Univariate analysis } \\
\text { p-value }\end{array}$} & \multicolumn{3}{|c|}{ Multivariate analysis } \\
\hline & & & HR & $95 \% \mathrm{Cl}$ & p-value \\
\hline \multicolumn{6}{|l|}{ Age } \\
\hline$<60$ years & $5.9(4.4-7.4)$ & 0.344 & & & \\
\hline$>60$ years & $6.0(5.3-6.7)$ & & & & \\
\hline \multicolumn{6}{|l|}{ Sex } \\
\hline Female & $6.0(4.9-7.1)$ & 0.428 & & & \\
\hline Male & $5.9(4.9-7.0)$ & & & & \\
\hline \multicolumn{6}{|l|}{ ECOG } \\
\hline $0-1$ & $6.2(4.8-7.5)$ & 0.007 & 1.487 & $1.064-2.076$ & 0.020 \\
\hline $2-4$ & $5.2(3.6-6.9)$ & & & & \\
\hline \multicolumn{6}{|l|}{ Stage } \\
\hline III & $8.7(6.6-10.7)$ & 0.001 & 1.595 & $1.145-2.222$ & 0.006 \\
\hline IV & $5.5(4.9-6.1)$ & & & & \\
\hline \multicolumn{6}{|l|}{ Smoking status } \\
\hline Positive & $6.0(5.1-6.8)$ & 0.808 & & & \\
\hline Negative & $5.9(4.7-7.2)$ & & & & \\
\hline \multicolumn{6}{|c|}{ Wood smoke exposure } \\
\hline Positive & $27.6(19.3-35.8)$ & 0.597 & & & \\
\hline Negative & $20.9(13.4-28.3)$ & & & & \\
\hline \multicolumn{6}{|l|}{ EGFR status } \\
\hline Positive & $7.5(6.2-8.8)$ & 0.297 & & & \\
\hline Negative & $5.5(4.9-6.0)$ & & & & \\
\hline \multicolumn{6}{|l|}{ Groups } \\
\hline Intermediate & $5.5(4.6-6.5)$ & 0.009 & 0.864 & $0.753-0.992$ & 0.039 \\
\hline High & $6.4(5.0-7.9)$ & & & & \\
\hline
\end{tabular}

Data are presented as median (95\% CI), unless otherwise stated. HR: hazard ratio; ECOG: Eastern Cooperative Oncology Group; EGFR: epidermal growth factor receptor. 
TABLE 5 Factors associated with overall survival

\begin{tabular}{|c|c|c|c|c|c|}
\hline & \multirow{2}{*}{$\begin{array}{l}\text { Overall survival } \\
\text { months }\end{array}$} & \multirow{2}{*}{$\begin{array}{l}\text { Univariate analysis } \\
\text { p-value }\end{array}$} & \multicolumn{3}{|c|}{ Multivariate analysis } \\
\hline & & & HR & $95 \% \mathrm{Cl}$ & p-value \\
\hline \multicolumn{6}{|l|}{ Age } \\
\hline$<60$ years & $20.1(15.1-25.0)$ & 0.113 & & & \\
\hline$>60$ years & $14.2(7.3-21.1)$ & & & & \\
\hline \multicolumn{6}{|l|}{ Sex } \\
\hline Female & 22.7 (16.8-28.5) & 0.012 & 1.588 & $0.860-2.93$ & 0.139 \\
\hline Male & $14.2(11.9-16.5)$ & & & & \\
\hline \multicolumn{6}{|l|}{ ECOG } \\
\hline $0-1$ & $20.7(16.3-25.2)$ & $<0.001$ & 2.04 & $1.168-3.56$ & 0.012 \\
\hline $2-4$ & $10.6(8.7-12.5)$ & & & & \\
\hline \multicolumn{6}{|l|}{ Stage } \\
\hline III & $24.3(8.5-40.1)$ & 0.029 & 2.16 & $1.08-4.30$ & 0.029 \\
\hline IV & $15.0(9.6-20.4)$ & & & & \\
\hline \multicolumn{6}{|l|}{ Smoking status } \\
\hline Positive & $24.3(19.4-29.2)$ & 0.046 & 1.204 & $0.661-2.19$ & 0.544 \\
\hline Negative & $14.4(12.3-16.5)$ & & & & \\
\hline \multicolumn{6}{|c|}{ Wood smoke exposure } \\
\hline Positive & $15.2(9.7-20.7)$ & 0.921 & & & \\
\hline Negative & $20.4(13.8-27.1)$ & & & & \\
\hline \multicolumn{6}{|l|}{ EGFR status } \\
\hline Positive & $27.3(11.5-43.1)$ & 0.027 & 1.999 & $1.142-3.498$ & 0.015 \\
\hline Negative & $14.8(11.3-18.3)$ & & & & \\
\hline \multicolumn{6}{|l|}{ Groups } \\
\hline Intermediate & $16.8(11.8-21.8)$ & 0.023 & 0.804 & $0.624-1.036$ & 0.037 \\
\hline High & $25.0(15.4-34.6)$ & & & & \\
\hline
\end{tabular}

Data are presented as median (95\% CI), unless otherwise stated. HR: hazard ratio; ECOG: Eastern Cooperative Oncology Group; EGFR: epidermal growth factor receptor.

We found that lepidic-predominant and acinar-predominant ADC (intermediate-grade group), had a poorer overall survival compared with papillary, micropapillary and solid ADC (high-grade group). Contrary to our results, several research groups have found a better survival in patients within intermediategrade group in early disease; the differences may be due to better response and better PFS to chemotherapy in our patients with high-grade histology. In this regard, various studies have found that the response rate in response to chemotherapy of patients with bronchioalveolar (bronchioalveolar carcinoma) histology, actually called lepidic ADC, is low $(6-14 \%)$ [13, 14]. Similarly, it has been reported that patients not selected for the presence of EGFR mutation and with bronchioalveolar carcinoma, have a lower response to EGFR-TKIs versus EGFR-mutated patients (responses between 11-22\%) [15]. In our case, only a few patients were treated with EGFR-TKIs, which in turn may also contribute to our patients' lower survival. An analysis of Ki-67 in lung tumours showed that high differentiation promotes cell proliferation significantly; tumours with higher bronchioalveolar carcinoma component have lower expression of Ki-67, reflecting decreased proliferation, which could be related to a lower rate of response to chemotherapy [16]. It is also important to mention that, unlike early disease, on the advanced disease the specimens to analyse were TruCut biopsies rather than resection specimens and probably could influence these differences.

A German study recently found that the overall survival varied significantly between groups, with the lowgrade group (lepidic-predominant $\mathrm{ADC}$ ) having 78.5 months, intermediate-grade (acinar-predominant ADC, and papillary-predominant) 48.9 months, and high-grade (micropapillary-predominant and solidpredominant ADC) 58.1 months [8]. In this cohort, patients with papillary-predominant ADC had a poor prognosis, similar to those with micropapillary and solid-predominant ADC; this finding may suggest that predominant pattern groups are predictors of survival independent of adjuvant chemoradiotherapy [8]. In the US, Australian and German studies it was suggested that identifying poor prognostic groups such as solid-predominant, micropapillary-predominant and papillary-predominant ADC is important, because patients with these tumours may be candidates for adjuvant therapy [6-8]. In chemotherapy-naïve patients like those in the US, Australian, Japanese and some in the German cohorts, prognosis was poor for highgrade ADCs, contrary to what we found in our cohort in which we observed a better prognosis in the 
high-grade ADCs [6-9]. However, the German group observed that patients from the high-grade group, in particular patients with papillary-predominant ADC that received chemotherapy, had a better prognosis when compared with the others patterns, thus they suggested that the prognostic significance of papillarypredominant ADC morphology might differ in chemotherapy-naïve patients and patients having received adjuvant therapy [8]. Moreover, similar to our results, the German report found that patients with lepidicpredominant $\mathrm{ADC}$ that received chemotherapy had worse prognosis than patients with solid-predominant ADC [8]. Our data and the data of Germany study indicate that there might be different pattern-dependant responses to chemotherapy. These previous studies associated the prognosis with the histology subtype in early disease, specifically reports from German and Japan $[8,17,18]$. These authors found that the patients with papillary ADC have a similar overall survival with patients harbouring micropapillary and solid predominant ADC. Also, in our patients with advanced disease we found a similar behaviour between the papillary, micropapillary and solid predominant ADCs, for this reason we included papillary histology in a high-grade group. The papillary ADCs maybe have a heterogeneous morphology and could be more aggressive.

While it has been reported that EGFR mutations are more common in lepidic-predominant, papillarypredominant and micropapillary-predominant ADCs, and less frequent in the solid-predominant subtype [19], we did not find any association between the presence of activating EGFR mutations and the IASLC/ ATS/ERS classification. We found a trend in relation to the frequency of EGFR mutations in lepidic, acinar and papillary patterns compared with micropapillary and solid patterns; in addition, patients with intermediate-grade tumours had a higher frequency of common mutations in EGFR (Del Ex19 and L858R) compared with high-grade tumours. We believe this has an important clinical significance since several studies have shown that the response to EGFR-TKIs is better for patients with common mutations [20]. In the Japanese study, the presence of EGFR mutation did not show association with any histological type; however, EGFR mutation were more frequently found in nonmucinous lepidic ADCs [9].

Although our study attempted to investigate the prognosis of patients in advanced disease, in all of our patients the diagnosis is made by Tru-Cut, we understand that, unlike in resected early stage disease, having a small sample may "blind" the pathologist to other components of patterns in the rest of the primary or metastatic tumour, and this may be a limitation of our study. Another limitation is that only $64.2 \%$ of the patients were genotyped for EGFR due to insufficient sample of tissue.

In summary, this is the first study to correlate the new classification of ADC in advanced disease. Our findings suggest that the prognostic differences found with the new classification in early disease may not applicable to patients with advanced or metastatic disease. Unlike in early stages, patients with advanced disease and high-grade ADC according to the new classification, have longer overall survival compared with intermediate-grade ADC, probably due to better response to chemotherapy. For this reason, we suggested that is essential to achieve a maximum histological characterisation according to the new IASLC/ATS/ERS so that there is clinical benefit in patients with NSCLC advanced disease.

\section{References}

Jemal A, Siegel R, Xu J, et al. Cancer statistics, 2010. CA Cancer J Clin 2010; 60: 277-300.

Arrieta O, Guzmán-de Alba E, Alba-López LF, et al. Consenso nacional de diagnóstico y tratamiento del cáncer de pulmón de células no pequeñas [National Consensus on the diagnosis and treatment of non-small cell lung cancer]. Rev Inv Clin 2013; 65: supl., 1: S5-S64.

3 Ruíz-Godoy L, Rizo Rios P, Sánchez Cervantes F, et al. Mortality due to lung cancer in Mexico. Lung Cancer 2007; 58: 184-190.

4 Devesa SS, Bray F, Vizcaino AP, et al. International lung cancer trends by histologic type: male:female differences diminishing and adenocarcinoma rates rising. Int J Cancer 2005; 117: 294-299.

5 Travis WD, Brambilla E, Noguchi M, et al. International Association for the Study of Lung Cancer/American Thoracic Society/EuropeanRespiratory Society: international multidisciplinary classification of lung adenocarcinoma. J Thorac Oncol 2011; 6: 244-285.

6 Yoshizawa A, Motoi N, Riely GJ, et al. Impact of proposed IASLC/ATS/ERS classification of lung adenocarcinoma: Prognostic subgroups and implications for further revision of staging based on analysis of 514 stage I cases. Mod Pathol 2011; 24: 653-664.

7 Russell PA, Wainer Z, Wright GM, et al. Does lung adenocarcinoma subtype predict patient survival? A clinicopathologic study based on the new International Association for the Study of Lung Cancer/American Thoracic Society/European Respiratory Society international multidisciplinary lung adenocarcinoma classification. J Thorac Oncol 2011; 6: 1496-1504.

8 Warth A, Muley T, Meister M, et al. The novel histologic International Association for the Study of Lung Cancer/ American Thoracic Society/European Respiratory Society classification system of lung adenocarcinoma is a stageindependent predictor of survival. J Clin Oncol 2012; 30: 1438-1446.

9 Yoshizawa A, Sumiyoshi S, Sonobe M, et al. Validation of the IASLC/ATS/ERS lung adenocarcinoma classification for prognosis and association with EGFR and KRASgene mutations: analysis of 440 Japanese patients. $J$ Thorac Oncol 2013; 8: 52-61.

10 Travis WD, Brambilla E, Muller-Hermelink HK, et al. Eds. Pathology and Genetics: Tumours of the Lung, Pleura, Thymus and Heart. World Health Organization Classification of Tumors. Lyon, IARC Press, 2004. 
11 Travis WD, Brambilla E, Noguchi M, et al. International Association for the Study of Lung Cancer/American Thoracic Society/European Respiratory Society international multidisciplinary classification of lung adenocarcinoma. J Thorac Oncol 2011; 6: 244-285.

12 Azzoli CG, Temin S, Aliff T, et al. 2011 focused update of 2009 American Society of Clinical Oncology clinical practice guideline update on chemotherapy for stage iv non-small-cell lung cancer. J Clin Oncol 2011; 29: 3825-3831.

13 Miller VA, Hirsch FR, Johnson DH. Systemic therapy of advanced bronchioloalveolar cell carcinoma: challenges and opportunities. J Clin Oncol 2005; 23: 3288-3293.

14 Schiller JH, Harrington D, Belani CP, et al. Comparison of four chemotherapy regimens for advanced non-smallcell lung cancer. N Engl J Med 2002; 346: 92-98.

15 Miller VA, Hirsch FR, Johnson DH. Systemic therapy of advanced bronchioloalveolar cell carcinoma: challenges and opportunities. J Clin Oncol 2005; 23: 3288-3293.

16 Sarantopoulos GP, Gui D, Shintaku P, et al. Immunohistochemical analysis of lung carcinomas with pure or partial bronchioloalveolar differentiation. Arch Pathol Lab Med 2004; 128: 406-414.

17 Aida S, Shimazaki H, Sato K, et al. Prognostic analysis of pulmonary adenocarcinoma subclassification with special consideration of papillary and bronchioloalveolar types. Histopathol 2004; 45: 468-476.

18 Yokose T, Suzuki K, Nagai K, et al. Favorable and unfavorable morphological prognostic factors in peripheral adenocarcinoma of the lung $3 \mathrm{~cm}$ or less in diameter. Lung Can 2000; 29: 179-188.

19 Shim HS, Lee da H, Park EJ, et al. Histopathologic characteristics of lung adenocarcinomas with epidermal growth factor receptor mutations in the International Association for the Study of Lung Cancer/American Thoracic Society/European Respiratory Society lung adenocarcinoma classification. Arch Pathol Lab Med 2011; 135: 1329-1334.

20 Yang JC, Shih JY, Su WC, et al. Afatinib for patients with lung adenocarcinoma and epidermal growth factor receptor mutations (LUX-Lung 2): a phase 2 trial. Lancet Oncol 2012; 13: 539-548. 